\title{
Libro Alteraciones endocrinas del paciente hospitalizado
}

$\mathrm{L}$ as alteraciones endocrinas en el paciente hospitalizado representan un reto desde su diagnóstico y manejo por la complejidad de la enfermedad en sí y por las múltiples variables que deben integrarse para optimizar su estabilidad fisiológica. Un aspecto fundamental que hay que considerar en el paciente hospitalizado es la homeostasis endocrina, de la que dependen un sinnúmero de vías de señalización intracelulares e intercelulares que se traducen en la sincronización de funciones metabólicas y orgánicas.

En los últimos años se han logrado avances en el conocimiento no solamente de la función endocrina, sino también de su disfunción y en la presentación de nuevas entidades endocrinológicas en el paciente internado, que no son exclusivamente una curiosidad fisiopatológica, ya que impactan en el comportamiento clínico, en el pronóstico y en la rehabilitación de este grupo especial de enfermos.

De las descripciones iniciales de la ya clásica respuesta metabólica al trauma y de las fases de flujo se ha alcanzado un elevado grado de conocimiento en el aspecto molecular y clínico de la disfunción endocrina en los enfermos hospitalizados. Por este motivo y por su gran interés en poner al día al grupo médico encargado del manejo del paciente hospitalizado, la Asociación Colombiana de Endocrinología, Diabetes y Metabolismo desarrolló un esfuerzo conjunto, bajo el liderazgo del doctor Alejandro Pinzón Tovar, para producir este libro de texto en el que se exponen los temas de más actualidad en relación a esta interesante e innovadora rama de la medicina endocrinológica, la disfunción endocrina del paciente hospitalizado.

El fascinante mundo del conocimiento del paciente hospitalizado está avanzando; es trascendental lo que ahora sabemos de la respuesta endocrina y existe un panorama muy amplio de posibilidades terapéuticas. Sin lugar a dudas, esta obra enriquece el conocimiento del estudio y el tratamiento de este complejo sistema.
El libro Alteraciones endocrinas en el paciente hospitalizado, tiene 49 capítulos agrupados en secciones, trata los recientes descubrimientos en el estudio de todos los cambios que se suceden en los pacientes hospitalizados con enfermedades endocrino-metabólicas, la utilidad en el diagnóstico al identificar esos cambios y las posibilidades terapéuticas.

De seguro, la lectura de este libro sembrará entre todos los interesados en medicina hospitalaria la semilla que al dar fruto sentará las bases de esta interesante faceta a la que con frecuencia no se le da su justa dimensión y trascendencia. Por otro lado, estoy seguro de que alertará y concientizará al clínico sobre las entidades endocrinológicas, así como sus manifestaciones y abordaje diagnóstico y terapéutico a las que se enfrenta cotidianamente el médico que trabaja en el ambiente hospitalario.

Con este libro de texto, la endocrinología colombiana se pone a la vanguardia al proponer un tema de gran actualidad y vigencia, en la que había vacíos en su diagnóstico y tratamiento.

La iniciativa del doctor Alejandro Pinzón Tovar merece admiración, reconocimiento y agradecimiento por parte de nuestra Asociación Colombiana de Endocrinología, Diabetes y Metabolismo y contó con el apoyo de médicos endocrinólogos y de especializaciones afines de todo nuestro territorio.

Finalmente, el libro Alteraciones Endocrinas en el Paciente Hospitalizado demuestra la gran fortaleza de nuestra asociación, que es la unión de todos nuestros endocrinólogos en la expresión del conocimiento.

\section{John Jairo Duque Ossman}

Médico Endocrinólogo

Miembro de Número de la Asociación Colombiana de Endocrinología, Diabetes y Metabolismo 